\title{
Monitoring of somatic cells in milk via laser analytical techniques for the early detection of mastitis
}

\author{
Z. Abdel-Salam • S. A. Attala • E. Daoud • \\ M. A. Harith
}

Received: 18 October 2014 / Revised: 3 December 2014 / Accepted: 5 January 2015 /

Published online: 22 January 2015

(C) INRA and Springer-Verlag France 2015

\begin{abstract}
Mastitis is an inflammatory infection of the mammary glands in dairy cattle, which causes a pronounced increase in milk somatic cell count accompanied by changes in milk elemental and molecular composition. The aim of the present study was to use different laser analytical techniques for fast, accurate, and easy diagnosis of mastitis. Both laser-induced breakdown spectroscopy (LIBS) and laser-induced fluorescence (LIF) techniques were used for this purpose. The study was performed on milk samples taken from 150 Holstein cows. The results obtained by LIBS showed that in healthy milk (milk from uninfected cows), the intensities of calcium spectral lines were clearly higher than in mastitic milk samples. On the contrary, the intensities of the two sodium D-lines were higher in mastitic milk than in the healthy milk. A linear relationship exists between the sodium spectral line intensities and the average of corresponding somatic cell count (SCC). Adopting LIF, the fluorescence intensity was also found to correlate linearly with the SCC of milk samples. Comparing the two proposed laser analytical techniques with other conventional techniques showed that the proposed techniques are faster, much easier, costeffective, and can be used in situ to monitor somatic cells for diagnosis of mastitis.
\end{abstract}

Keywords Mastitis diagnosis $\cdot \mathrm{SCC} \cdot$ Sodium and calcium $\cdot$ Laser analytical techniques

Z. Abdel-Salam • M. A. Harith ( $\square)$

National Institute of Laser Enhanced Science, Cairo University, Giza 12613, Egypt

e-mail: mharithm@niles.edu.eg

S. A. Attala

Animal Production Department, Faculty of Agriculture, Cairo University, Giza, Egypt

E. Daoud

Agricultural Research Council, Food Technology Research Institute, Giza, Egypt 


\section{Introduction}

Mastitis is defined as an inflammation of the mammary gland and udder tissue which is most often due to bacterial infection. Usually, mastitis in dairy cows is diagnosed via estimating somatic cell counts (SCC) (Dohoo and Meek 1982; Fenlon et al. 1995) of the suspected milk sample. Mastitis has a direct impact on the produced milk composition. Reduction of milk yield and drastic decline in milk quality which includes significant changes in the protein and mineral content are the most obvious symptoms of mastitis. It is well known that bovine mastitis has negative dramatic effects on animal health. In addition, it is a risk factor for pregnancy loss in dairy cows (Jorge et al. 2012). Accordingly, it is considered to be the most periodic and costly disease in dairy industry. Using atomic absorption spectrophotometry technique showed that udder infection leads to an increase in sodium and chloride and a decrease in potassium content in cows' milk (Wegner and Stull 1978). On the other hand, analyzing milk samples from cows presenting mastitis symptoms revealed a remarkable decrease in calcium level (Batavani et al. 2007). An interesting study performed by Liu et al. (2009) on mastitic milk demonstrated that the SCC is directly proportional to the immunoglobulin (IgG) concentration. Different methods have been suggested for detection of mastitis in cattle, such as the California Mastitis Test (CMT), somatic cell counts (SCC), and electrical conductivity (EC) of milk (Lafi 2006; Norberg et al. 2004; Norberg 2005). However, developing in situ applicable diagnostic techniques for mastitis will facilitate the early detection of the disease to reduce its negative consequences as much as possible and to raise treatment success rates.

Elemental and molecular analyses of different types of samples via noninvasive laser analytical techniques such as laser-induced breakdown spectroscopy (LIBS) and laserinduced fluorescence (LIF) are currently preferred and privileged for in situ measurements with minimal or no sample preparation. Advanced LIBS and LIF compact and portable systems are now commercially available for numerous field applications. This is highly important, especially for field applications in animal production and dairy where it is very useful to perform measurements directly in the farms and avoid transportation of the samples to the laboratories. LIBS is a straightforward and easy elemental analysis technique that has many biological and medical applications. ElHussein et al. (2010) published an interesting in vitro study that used LIBS to identify and characterize some types of human malignancies. LIBS was also used by Yueh et al. (2009) for the analysis of chicken brain, lung, spleen, liver, kidney, and skeletal muscle. LIBS has also been used in other biological applications such as bacteria identification (Steven et al. 2007) and biological tissues mapping to follow up the distribution of certain elements (Sancey et al. 2014). Another interesting application for LIBS in biology is its use in evaluation of maternal and infant formula milk (Abdel-Salam et al. 2013). LIF which is normally used for detection of selective species and studying the structures of molecules has been recently exploited to estimate sperm count in buffalo semen samples (Abdel-Salam and Harith 2012). The aim of the present work is to adopt LIBS and LIF techniques for characterization of mastitic milk elementally and molecularly, respectively. The relative concentration of calcium and sodium represented by their spectral line intensities in the LIBS spectra of the milk samples will be correlated to the SCC. The fluorescence intensity of mastitic milk and healthy milk will be also correlated to the SCC in these samples. The final goal is to utilize LIBS and LIF 
as simple, fast, and inexpensive analytical techniques for the early detection of mastitis by monitoring somatic cells in milk samples.

\section{Materials and methods}

\subsection{Milk sample collection}

Aseptic milk samples were randomly collected from 150 Holstein cows kept near to the same conditions and weight in an accredited animal production farm at Salhia (about $100 \mathrm{~km}$ northeast of Cairo). The samples were randomly divided into two groups each of 75: one group was for LIBS experiments, and the other group was for LIF measurements. The samples were hygienically collected and properly stored at -20 ${ }^{\circ} \mathrm{C}$. A small portion of each sample was taken for somatic cell count. For LIBS measurements, the frozen samples were thawed at room temperature. Droplets of these samples $(0.5 \mathrm{~mL} /$ droplet $)$ were taken onto an ashless filter paper then left to be absorbed and expanded homogeneously in clean dry air for about $15 \mathrm{~min}$ before further LIBS analysis. One milliliter of each thawed sample was taken in a standard quartz cuvette with four transparent sides for conducting LIF experiments.

\subsection{Mastitis definition}

Milk samples from cows showing observable signs of mastitis were aseptically collected for further experimental investigations. Sometimes, mastitis symptoms could be undetectable visibly, with no abnormality in the milk, so somatic cell count (SCC) was considered as a decisive rule for selecting cows to determine whether a cow is infected with mastitis (Kramer et al. 2009; Miekley et al. 2012). A conventional cell counting system (Somacount 150, Bentley, USA) was used for counting somatic cells. In this system, a mixture of milk samples and a fluorescent dye was passed through a flow cell dispersing the globules to distinguish the stained DNA in the somatic cells. An aliquot part of the stained suspension was injected into a laminar stream of the carrier fluid. Then, the somatic cells were separated by the stream. When the stained cells were exposed to a laser beam, they produced fluorescent light pulses. The created light pulses were converted into electrical pulses via a photomultiplier tube. The pulses are sorted, counted, and translated onto a somatic cell counter via special software. A sample with somatic cell count $<300,000$ cells $/ \mathrm{mL}$ was classified as uninfected sample, and that with $\mathrm{SCC}>300,000$ cells $/ \mathrm{mL}$ was classified as infected sample. However, lower levels of SCC may be considered as disease identification depending on animal health standards in each geographical region (Pinto et al. 2013).

\subsection{LIBS arrangement}

In LIBS, a focused Q-switched laser pulses produce the so-called plasma plume onto the surface of the target to be analyzed. The plasma plume consists of a collection of ions and swirling electrons at enormously high temperature $(>6000 \mathrm{~K})$. The light emitted from the cooling down plasma plume is collected by a proper lens system or optical fiber and fed to a suitable spectrometer equipped with a detector for dispersion 
and detection of the plasma emission light. The finally obtained spectrum includes the characteristic spectral lines of the elements existing in the plasma plume and consequently in the target material. Detailed description of the LIBS technique is found in a number of books and review papers (Miziolek et al. 2006; Singh and Thakur 2007; Singh et al. 2009; Kaiser et al. 2012). In the present work, we used a typical LIBS setup in which a Q-switched Nd:YAG laser (BRIO, Quantel, France) produces laser pulses of $50 \mathrm{~mJ} /$ pulse at a wavelength $\lambda=1064 \mathrm{~nm}$ and pulse width $5 \mathrm{~ns}$ at a repetition rate $1 \mathrm{~Hz}$. The laser pulses were focused onto the surface of the sample under investigation using a planoconvex quartz lens of focal length $10 \mathrm{~cm}$. The sample was fixed on an X-Y micrometric translational stage. The light emitted from the laser-produced plasma plume was collected by a fused-silica optical fiber with a diameter of $600 \mu \mathrm{m}$ held at a distance of $2 \mathrm{~cm}$ above the plasma at an angle of $30^{\circ}$ with respect to the target surface. Then, the collected plasma emission was fed via the optical fiber to the entrance slit of an echelle spectrometer (Mechelle 7500, Multichannel, Sweden) equipped with an ICCD camera as a detector (DiCAM-Pro, PCO, Computer optics, Germany). To avoid electronic interference and jitters, the CCD intensifier high voltage was triggered optically at a typical optimized delay time of $2000 \mathrm{~ns}$ with integration time $2000 \mathrm{~ns}$. Spectra were collected from five fresh spots on the milk sample on an ashless filter paper. Spectra of five laser shots were collected from five milk spots, and a final average of 25 spectra were recorded and analyzed. Spectra processing and spectral line identification have been performed by the commercial software 2D- and 3D-Gram/32.

\subsection{LIF arrangement}

Laser-induced fluorescence (LIF) is the other laser analytical technique used in the present work. In LIF, atoms and molecules can be excited by absorption of laser photons of a specific wavelength, then emit light at a longer wavelength to get rid of the previously absorbed optical radiation energy. The emitted light during the de-excitation (wavelength and intensity) was measured to identify species under investigation.

In the present measurements, a typical LIF setup described in details elsewhere (Abdel-Salam and Harith 2012) was used. A diode-pumped solid-state (DPSS) laser [Changchun New Industries Optoelectronics Tech Co. Ltd., (China)] which emits $40 \mathrm{~mW}$ laser light of wavelength $\lambda=405 \mathrm{~nm}$ was used as an excitation source. The laser light was focused onto the surface of a quartz cuvette containing the milk sample, and the fluorescence was perpendicularly collected and fed to a compact spectrometerdetector system (USB2000 FLG, Ocean Optics, FL, USA) via an optical fiber. The obtained fluorescence spectra were recorded and processed using SpectraSuite software (Ocean Optics, FL, USA). Data processing and results presentation were accomplished by Origin 8.5 software (OriginLab Corp., MA, USA).

\section{Results}

\subsection{LIBS analysis}

To follow up the relative concentration of calcium and sodium in the samples under investigation, the intensity of four calcium spectral lines and two sodium lines 
normalized for the strong carbon line at $247.8 \mathrm{~nm}$ have been studied. For calcium, the elected emission lines were at 428.3, 428.9, 430.8, and $431.9 \mathrm{~nm}$ while for sodium, the well-known doublet at 589.0 and $589.6 \mathrm{~nm}$ were elected. These lines have been selected because of being well resolved and spectral-interference free as shown in Fig. 1. LIBS spectra demonstrated that the intensity of calcium spectral lines in healthy milk samples with low SCC $\left(>300 \times 10^{3}\right.$ cells $\left./ \mathrm{mL}\right)$ is clearly higher than in mastitic samples with high SCC $\left(<300 \times 10^{3}\right.$ cells $\left./ \mathrm{mL}\right)$ as shown in Fig. 2a. On the contrary, the two sodium lines in the same spectra have high intensity in case of mastitic milk compared to the normal milk as depicted in Fig. 2 b.

To employ the increase of sodium ions and decrease of calcium ions in mastitic samples, the sum of the intensities of the most bright sodium lines in the LIBS spectra (namely sodium D-lines at 589.0 and $589.6 \mathrm{~nm}$ normalized for calcium line at $422.7 \mathrm{~nm}$ ) has been plotted against different values of SCC of the milk samples. Figure 3 depicts a linear relationship which demonstrated that a higher sodium/ calcium ratio is associated with a higher SCC in such samples.

\subsection{LIF analysis}

Figure 4 shows typical LIF spectra of different healthy and mastitic milk samples which have different somatic cell counts (SCC). The fluorescence emission intensity has been found to increase systematically with the SCC of the individual samples. The linearity of the relationship between the somatic cell count and the fluorescence intensity of different samples is shown in Fig. 5.

\section{Discussion}

Calcium and sodium have been chosen to be followed up in LIBS spectra of the samples under investigation in view of the previously published work by Harmon (Harmon 1994). In such reference, the authors found that in mastitic samples, calcium

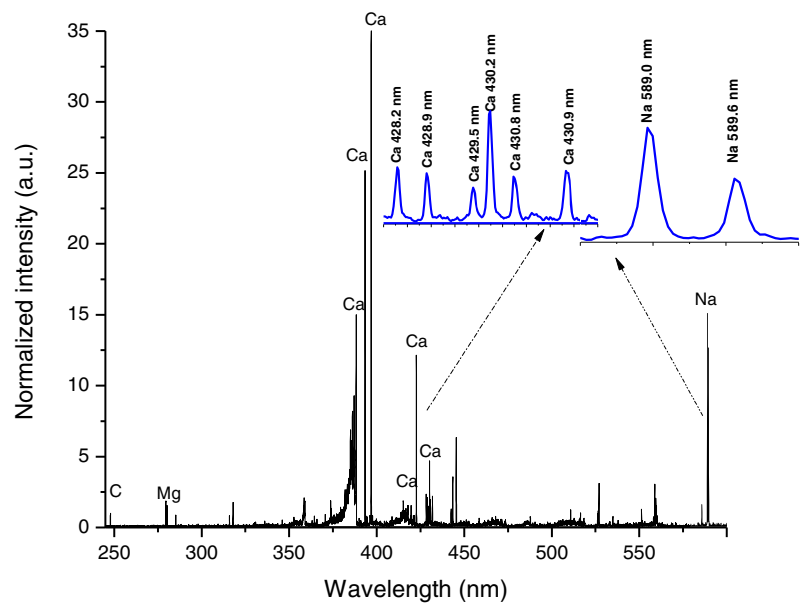

Fig. 1 A typical LIBS spectrum for infected milk sample. The insets show zoomed sections of the calcium and sodium spectral lines used in the analysis 

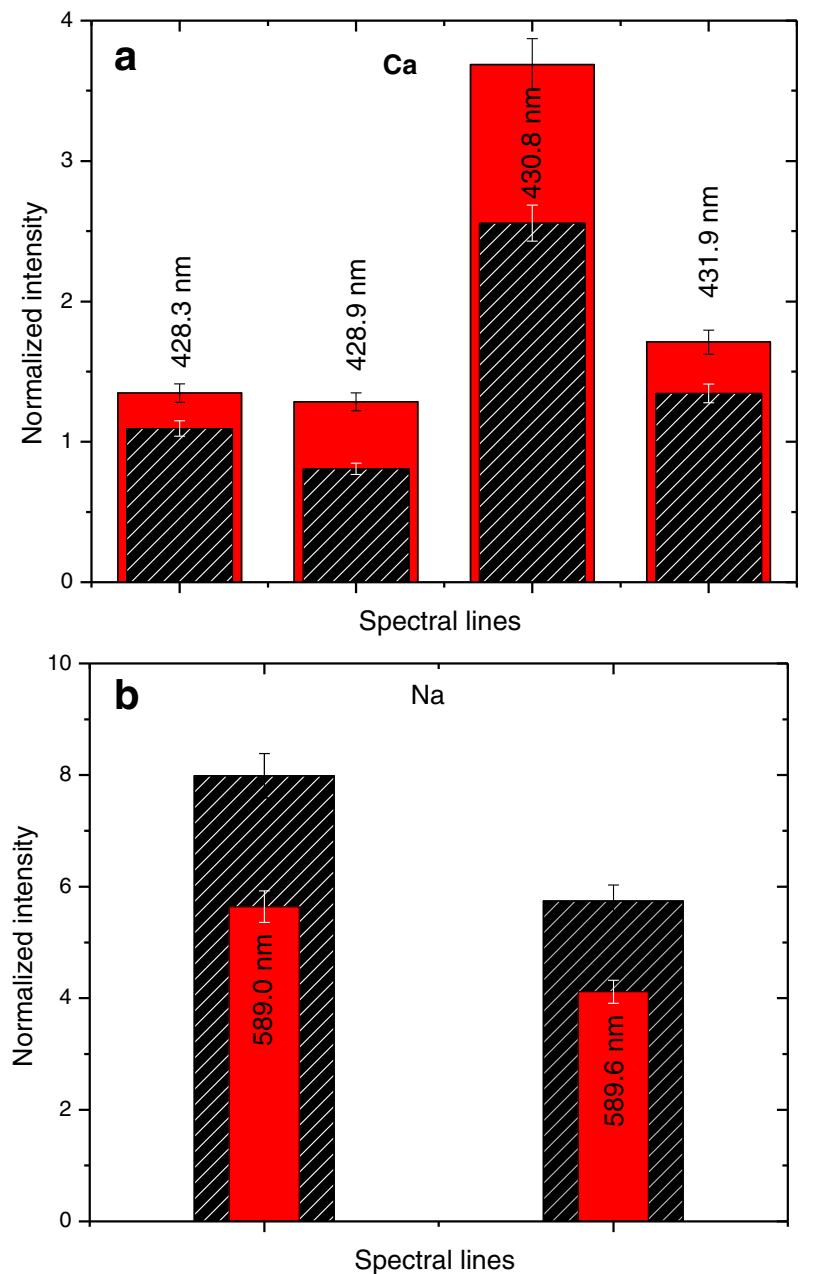

Fig. 2 The normalized intensity of four calcium spectral lines (a) and two sodium spectral lines (b), for mastitis samples (hatched) and normal milk samples (unhatched)

was $33 \%$ less, while sodium was $184 \%$ higher than that in the healthy milk. However, similar results on sodium have been also confirmed later by Batavani et al. 2007; they interpreted their results due to changes occurred in the ionic environment. The same authors and others (Kitchen 1981; Fernando et al. 1985; Vijayalakshmi et al. 2001; Bruckmaier et al. 2004) reported results relevant to the reduction in calcium levels in case of intramammary infections. On the other hand, Kitchen (1981) proved that the electric conductivity of mastitic milk is higher than that of healthy milk due to the increase in sodium ions in the infected sample. Our results clearly demonstrated that calcium emission lines have higher intensity in healthy milk with low SCC than in mastitic milk with higher SCC. On the contrary, sodium has high spectral line intensity in mastitic milk compared to the healthy milk. The linear relationship between the sum of the sodium D-lines intensities (normalized for Ca $422.7 \mathrm{~nm}$ line) and the SCC shown in Fig. 3 can be easily used for monitoring somatic cells in milk samples and 


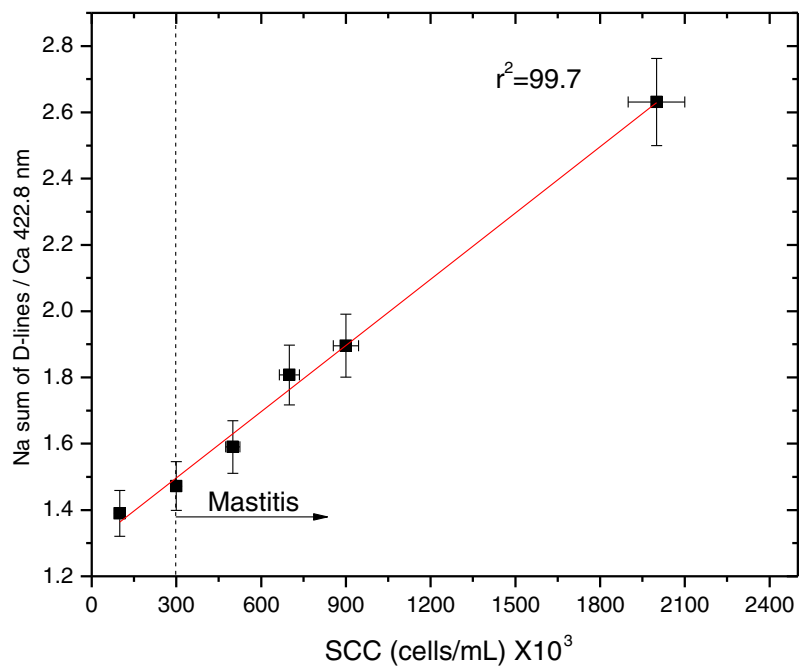

Fig. 3 Sum of the sodium D-lines at 589.0 and $589.6 \mathrm{~nm}$ normalized by Ca $422.8 \mathrm{~nm}$ line, versus different values of SCC count (cells $/ \mathrm{mL})$ in the milk samples

consequently detecting mastitis. Finding out the normalized sum of the sodium D-lines in the LIBS spectrum of any milk sample leads to the estimation of the corresponding average SCC from the obtained calibration curve. It is worthy to mention that the choice of $\mathrm{Na}$ and $\mathrm{Ca}$ lines with similar high energy levels for both elements (which is almost the case for the lines used) could reduce the influence of matrix effects related to the plasma temperature, and then, this ratio measurements should become relatively insensitive to the experimental fluctuations (which are quite relevant for in situ analysis). On the other hand, with such line configuration (i.e., same high energy level), the relative line intensity ratio $\mathrm{Na} / \mathrm{Ca}$ becomes directly proportional to the relative concentration $\mathrm{Na} / \mathrm{Ca}$, and only one calibrated sample can turn qualitative results to quantitative.

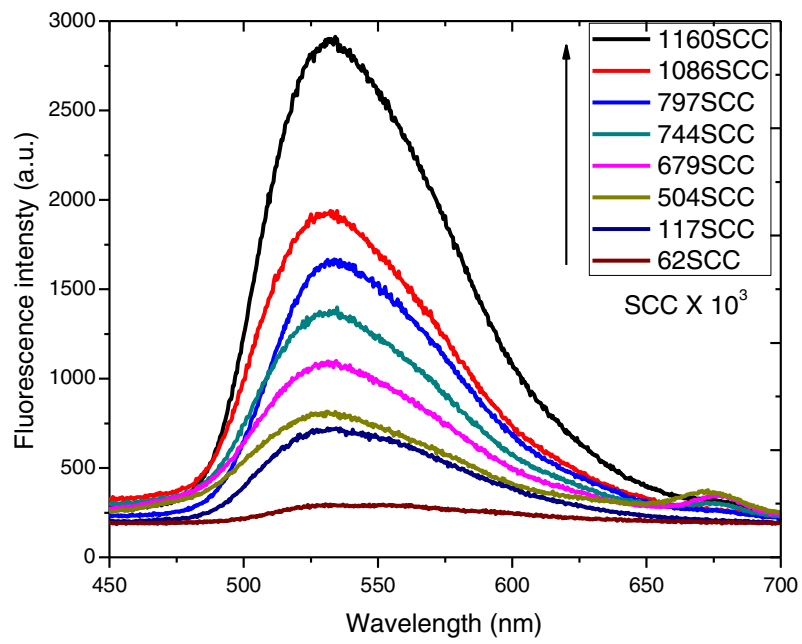

Fig. 4 Typical LIF spectra of different normal and mastitis milk samples of different SCC (cells/mL) 


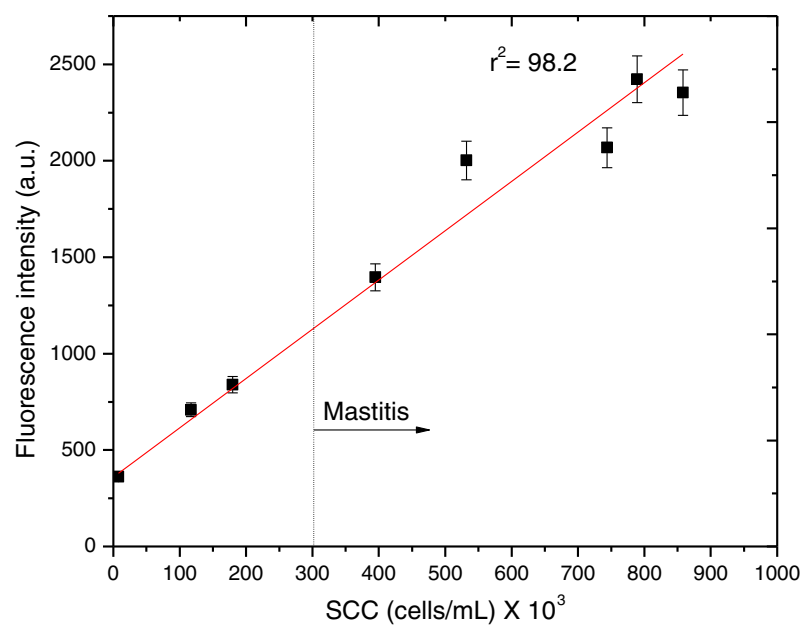

Fig. 5 The relation between the fluorescence intensity values and the SCC (cells $/ \mathrm{mL}$ )

The second part in the present study was conducted to demonstrate the feasibility of using LIF as a simple diagnostic technique for mastitis in dairy cattle. As mentioned above, it is widely known that milk SCC is commonly serving as an indicator of the incidence of mastitis in dairy cows (Dohoo and Meek 1982; Fenlon et al. 1995). Optical excitation of the samples, via laser light of wavelength $405 \mathrm{~nm}$, results in a fluorescence emission band extending from 475 to up to $650 \mathrm{~nm}$ peaking at $530 \mathrm{~nm}$ as shown in Fig. 4. The increase in the fluorescence intensity with increasing SCC can be interpreted in view of the high level of immunoglobulins (IgG) in the infected samples (Liu et al. 2009). Previous measurements on bovine colostrum (first milking postpartum) which contains high levels of IgG (Mainer et al. 2000; Gapper et al. 2007; Abdel-Salam et al. 2014) demonstrated that IgG has a fluorescence peak at $570 \mathrm{~nm}$. The deconvolution of mastitic fluorescence peak revealed that the emission peak consists of two peaks that are almost

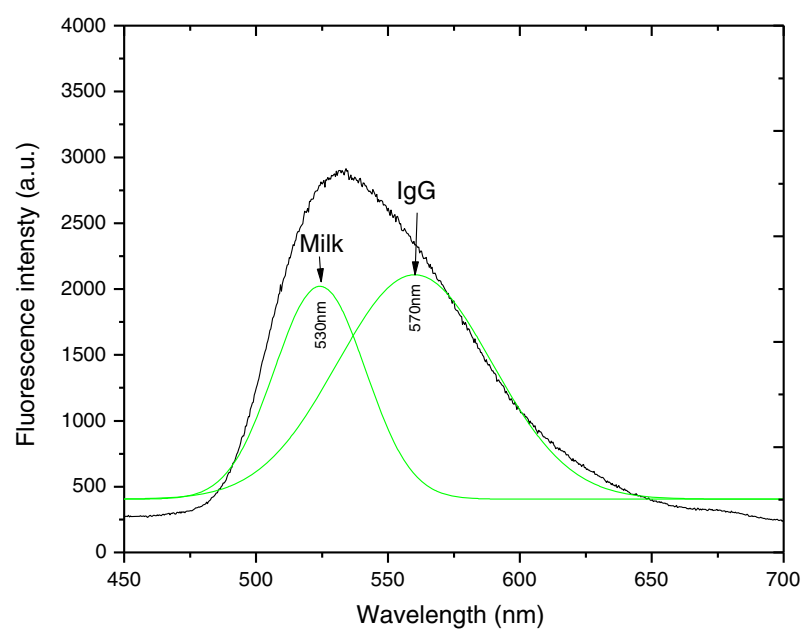

Fig. 6 Deconvolution of the fluorescence peak of an infected sample (black peak) showing the contribution of the IgG fluorescence with respect to the normal milk contribution 
completely superimposed (the milk peak at $530 \mathrm{~nm}$ and the $\mathrm{IgG}$ peak at $570 \mathrm{~nm}$ as shown in Fig. 6). This affirms that the peak at $570 \mathrm{~nm}$ in the mastitis samples corresponds to the increase in the IgG as a natural immune reaction against the bacterial infection.

In order to obtain quantitative results, it is essential to calibrate the used LIF system, just as any other spectrochemical analytical technique. In this work, the samples used for constructing the calibration curves were milk samples with known SCC values. The linearity of the relationship between the fluorescence intensity and the SCC $\left(r^{2}=98.2\right)$ demonstrates the feasibility of using LIF for the estimation of somatic cell count value in any unknown milk sample and consequently diagnosis of mastitis by adopting the relevant calibration curve. Because one of the major goals of this work was to introduce a simple and portable technique for diagnosis of mastitis, we excluded using hyphenated laser analytical techniques such as LIBS-Raman spectroscopy and LIF-optoacoustic spectrometry. Even the combined use of LIBS and LIF techniques is not recommended because of the complexity of the setup in such case. It is worth to mention that the time duration of a single sample measurement via LIBS or LIF is almost $10 \mathrm{~min}$ or less, requiring no sample preparation. Conventional techniques for SCC require 2 to $3 \mathrm{~h}$ for single sample measurement, besides requiring sample preparation and transportation to the laboratory.

\section{Conclusion}

In conclusion, the results reported in this paper show that using laser analytical techniques may provide new diagnostic possibilities for bovine mastitis. Using LIBS demonstrated that there is a positive correlation between SCC and sodium content in the investigated samples. A similar linear correlation also exists between SCC and the fluorescence intensity of the samples due to the increase of immunoglobulin concentration as an immune reaction. This work presents successful analytical techniques, namely LIBS and LIF as noninvasive, fast, and inexpensive methods for the early detection of mastitis. In addition, it is feasible to adopt both techniques for in situ measurements, i.e., in animal production farms as simple and fast diagnostic methods for early identification of infection instead of using lengthy and expensive conventional techniques. Although both LIBS and LIF are reliable and portable techniques, LIF equipment is simpler and cheaper which may make its usability more widely.

Conflict of interest Z. Abdel-Salam, S. A. Attala, E. Daoud, and M.A. Harith declare that they have no conflict of interest.

Statement of human and animal rights This article does not contain any studies with human or animal subjects performed by any of the authors.

\section{References}

Abdel-Salam ZA, Harith MA (2012) Laser spectrochemical characterization of semen. Talanta 99:140-145

Abdel-Salam ZA, Al Sharnoubi J, Harith MA (2013) Qualitative evaluation of maternal milk and commercial infant formulas via LIBS. Talanta 115:422-426 
Abdel-Salam ZA, Abdel Ghany S, Harith MA (2014) Evaluation of Immunoglobulins in bovine colostrum using laser induced fluorescence. Talanta 129:15-19

Batavani RA, Asri S, Naebzadeh H (2007) The effect of subclinical mastitis on milk composition in dairy cows. Iran Journal Veterinary Research. Univ Shiraz 8:205-211

Bruckmaier RM, Ontsouka CE, Blum JW (2004) Fractionized milk composition in dairy cows with subclinical mastitis. Vet Med Czech 49:283-290

Dohoo IR, Meek AH (1982) Somatic cell counts in bovine milk. Vet J 23:119-125

El-Hussein A, Kassem AK, Ismail H, Harith MA (2010) Exploiting LIBS as a spectrochemical analytical technique in diagnosis of some types of human malignancies. Talanta 82:495-501

Fenlon DR, Logue DN, Gunn J, Wilson J (1995) A study of mastitis bacteria and herd management practices to identify their relationship to high somatic cell counts in bulk tank milk. Br Vet J 151:17-25

Fernando RS, Spahr SI, Jester EH (1985) Comparison of electrical conductivity of milk with other indirect methods for detection of subclinical mastitis. J Dairy Sci 68:449-456

Gapper LW, Copestake EJ, Otter DE, Indyk HE (2007) Analysis of bovine immunoglobulin G in milk, colostrum and dietary supplements: a review. Anal Bioanal Chem 89:93-109

Harmon RJ (1994) Mastitis and genetic evaluation for somatic cell count. J Dairy Sci 77:2103-2112

Jorge AH, Carlos AR, Fabio SL, Jose EPS (2012) Observed and expected combined effects of clinical mastitis and low body condition on pregnancy loss in dairy cows. Theriogenology 77:115-121

Kaiser J, Novotný K, Martin M, Hrdlička A, Malina R, Hartl M, Adam V, Kizek R (2012) Trace elemental analysis by laser-induced breakdown spectroscopy biological applications. Surf Sci Rep 67:233-243

Kitchen BJ (1981) Review of progress of dairy science: bovine mastitis: milk compositional changes and related diagnostic tests. J Dairy Res 48:167-188

Kramer E, Cavero D, Stamer E, Krieter J (2009) Mastitis and lameness detection in dairy cows by application of fuzzy logic. Livest Sci 125:92-96

Lafi SQ (2006) Use of somatic cell counts and California mastitis test results from udder halves milk samples to detect subclinical intramammary infection in Awassi sheep. Small Ruminant Res 62:83-86

Liu GL, Wang JQ, Bu OP, Cheng JP, Zhang CG, Wei JY, Zhou LY, Zhou ZF, HU H, Dong XL (2009) Factors affecting the transfer of immunoglobulin G1 into the milk of Holstein cow. Vet J 182:79-85

Mainer G, Perez MD, Sanchez L, Puyol P, Millan MA, Dominguez JM, Ena E, Calvo M (2000) Concentration of bovine immunoglobulins throughout lactation and effect of sample preparation on their determination. Milchwissenschaft 55:613-617

Miekley B, Traulsen I, Krieter J (2012) Detection of mastitis and lameness in dairy cows using wavelet analysis. Livest Sci 148:227-236

Miziolek A, Palleschi V, Schechter I (2006) Laser-induced breakdown spectroscopy (LIBS) fundamentals and applications. Cambridge University Press, New York

Norberg E (2005) Electrical conductivity of milk as a phenotypic and genetic indicator of bovine mastitis: a review. Livest Prod Sci 96:129-139

Norberg E, Hogeveen H, Korsgaard IR, Friggens NC, Sloth KH, Løvendahl P (2004) Electrical conductivity of milk: ability to predict mastitis status. J Dairy Sci 87:1099-1107

Pinto G, Caira S, Nicolai MA, Mauriello R, Guollo M, Pirisi A, Piredda G, Chianese L, Addeo F (2013) Proteolysis and partial dephosphorylation of casein are affected by high somatic cell counts in sheep milk. Food Res Int 53:510-521

Sancey L, Motto-Ros V, Busser B, Kotb S, Benoit JM, Piednoir A, Lux F, Tillement O, Panczer G, Yu J (2014) Laser spectrometry for multi-elemental imaging of biological tissues. Sc Rep 4(6065):1-7

Singh JP, Thakur SN (2007) Laser-induced breakdown spectroscopy. Elsevier, Amsterdam

Singh VK, Rai AK, Rai PK, Jindal PK (2009) Prospects for laser-induced breakdown spectroscopy for biomedical applications: a review. Lasers Med Sci 24:749-759

Steven JR, Diedrich J, Palchaudhuri S (2007) Identification and discrimination of Pseudomonas aeruginosa bacteria grown in blood in bile by laser induced breakdown spectroscopy. Spectrochim Acta B 62:11691176

Vijayalakshmi P, Prathaban S, Dhanapalan P (2001) Comparative study on the efficacy of diagnostic tests in the field diagnosis of bovine mastitis. India Vet J 78:4-6

Wegner TN, Stull JW (1978) Relation between mastitis test score, mineral composition of milk and blood electrolyte profiles in Holstein cows. J Dairy Sci 61:1577-1579

Yueh FY, Zheng HP, Singh JP, Burgess S (2009) Preliminary evaluation of laser-induced breakdown spectroscopy for tissue classification. Spectrochim Acta B 64:1059-1067 